\title{
Elemental analysis of tetrahydrocannabinol and nicotine e-liquids related to EVALI
}

Kevin M. Kubachka ${ }^{1 *}$ and Robert A. Wilson ${ }^{1}$

1) U.S. Food and Drug Administration, Forensic Chemistry Center, Cincinnati, OH 45237, USA

\section{* Corresponding Author}

Keywords: Toxic Metals, e-liquids, vaping, EVALI 


\section{Supplemental Material}

\begin{tabular}{|c|c|c|c|c|c|c|c|c|c|c|c|c|c|c|c|}
\hline$\#$ & $\mathrm{Cr}$ & Mn & Co & $\mathrm{Ni}$ & $\mathrm{Cu}$ & $\mathrm{Zn}$ & As & Mo & Cd & Sn & Sb & Pt & $\mathrm{Au}$ & $\mathrm{TI}$ & $\mathrm{Pb}$ \\
\hline 1 & $0.348 \pm 0.014$ & 0.030 & ND & $8.79 \pm 0.20$ & $93.9 \pm 1.2$ & $60.1 \pm 3.4$ & 0.010 & 0.019 & 0.015 & $0.359 \pm 0.014$ & 0.016 & ND & ND & ND & $6.50 \pm 0.22$ \\
\hline 2 & ND & ND & ND & 0.30 & ND & ND & ND & ND & ND & 0.011 & 0.027 & ND & ND & ND & 0.034 \\
\hline 3 & ND & 0.024 & ND & $102 \pm 6$ & $2.27 \pm 0.07$ & ND & ND & 0.025 & ND & 0.019 & ND & ND & ND & ND & $0.903 \pm 0.036$ \\
\hline 4 & ND & ND & ND & $0.29 \pm 0.57$ & ND & ND & ND & ND & 0.002 & ND & ND & 0.022 & ND & ND & 0.029 \\
\hline 5 & ND & ND & ND & ND & ND & ND & ND & ND & ND & ND & ND & ND & ND & ND & 0.011 \\
\hline 6 & ND & ND & ND & $53 \pm 47$ & $150 \pm 170$ & $120 \pm 130$ & 0.028 & ND & 0.012 & $1.9 \pm 2.3$ & 0.059 & ND & $0.93 \pm 0.11$ & ND & $11 \pm 10$ \\
\hline 7 & ND & ND & $0.177 \pm 0.008$ & $0.36 \pm 0.41$ & $1.05 \pm 2.11$ & 2.9 & ND & ND & ND & $0.111 \pm 0.070$ & ND & ND & ND & ND & $3.75 \pm 0.17$ \\
\hline 8 & $3.89 \pm 0.55$ & $0.495 \pm 0.087$ & $0.041 \pm 0.004$ & $16.2 \pm 2.9$ & $34.0 \pm 1.9$ & $25.3 \pm 2.5$ & 0.008 & ND & 0.003 & $0.578 \pm 0.079$ & 0.036 & 0.007 & ND & ND & $2.35 \pm 0.23$ \\
\hline 9 & ND & ND & $0.107 \pm 0.024$ & $0.8 \pm 1.3$ & $1.2 \pm 1.9$ & ND & ND & ND & ND & $0.109 \pm 0.060$ & ND & ND & ND & ND & $3.0 \pm 9.0$ \\
\hline 10 & ND & ND & $0.108 \pm 0.028$ & 0.21 & ND & ND & ND & ND & ND & 0.060 & ND & ND & 0.027 & ND & $0.14 \pm 0.20$ \\
\hline 11 & ND & ND & $0.814 \pm 0.280$ & $5.4 \pm 5.3$ & $13 \pm 18$ & $18 \pm 13$ & ND & ND & ND & $1.12 \pm 0.70$ & ND & ND & 0.072 & ND & $5.0 \pm 9.8$ \\
\hline 12 & ND & ND & ND & $2.33 \pm 0.15$ & $0.16 \pm 0.15$ & ND & ND & ND & ND & 0.015 & ND & ND & ND & ND & $0.77 \pm 1.63$ \\
\hline 13 & ND & ND & $0.273 \pm 0.045$ & $1.3 \pm 1.4$ & 0.5 & ND & ND & ND & 0.017 & $0.496 \pm 0.088$ & ND & ND & ND & ND & $1.7 \pm 5.4$ \\
\hline 14 & ND & ND & $0.087 \pm 0.002$ & 0.10 & 0.2 & 2.5 & ND & ND & ND & 0.070 & ND & ND & ND & ND & $3.9 \pm 9.8$ \\
\hline 15 & ND & $0.164 \pm 0.008$ & $0.025 \pm 0.011$ & $477 \pm 21$ & $35.7 \pm 2.2$ & $32.1 \pm 0.5$ & ND & ND & 0.006 & $0.846 \pm 0.005$ & 0.027 & ND & 0.068 & 0.038 & $5.14 \pm 0.35$ \\
\hline 16 & ND & ND & ND & $0.713 \pm 0.099$ & ND & ND & ND & ND & ND & ND & ND & ND & ND & ND & $3.5 \pm 9.3$ \\
\hline 17 & ND & ND & $0.109 \pm 0.005$ & $3.48 \pm 0.18$ & $8.93 \pm 0.33$ & 5.9 & ND & 0.022 & ND & $0.407 \pm 0.033$ & ND & ND & ND & ND & $2.64 \pm 0.62$ \\
\hline 18 & ND & ND & $0.098 \pm 0.009$ & 0.10 & ND & ND & ND & ND & ND & 0.044 & ND & ND & ND & ND & $0.14 \pm 0.47$ \\
\hline 19 & ND & ND & 0.016 & ND & ND & ND & ND & 0.022 & ND & 0.013 & ND & ND & ND & ND & 0.013 \\
\hline 20 & ND & ND & $0.061 \pm 0.008$ & ND & ND & ND & ND & ND & ND & 0.045 & ND & ND & ND & ND & $0.100 \pm 0.031$ \\
\hline 21 & ND & $0.120 \pm 0.028$ & ND & 0.40 & ND & ND & ND & ND & ND & $0.137 \pm 0.338$ & ND & ND & ND & 0.041 & $2.6 \pm 7.2$ \\
\hline 22 & ND & ND & ND & $1.02 \pm 0.19$ & ND & ND & ND & ND & 0.009 & 0.067 & ND & ND & ND & ND & 0.012 \\
\hline 23 & ND & ND & ND & $0.67 \pm 0.98$ & 0.5 & ND & ND & 0.020 & $0.033 \pm 0.101$ & 0.041 & ND & ND & ND & ND & $0.102 \pm 0.040$ \\
\hline 24 & ND & ND & ND & $2.31 \pm 0.69$ & 0.9 & ND & 0.047 & 0.024 & ND & 0.025 & ND & ND & ND & 0.022 & $0.241 \pm 0.043$ \\
\hline 25 & ND & $0.083 \pm 0.235$ & $0.133 \pm 0.001$ & 0.19 & 0.4 & ND & ND & ND & ND & $0.33 \pm 0.13$ & ND & ND & ND & ND & $0.23 \pm 0.25$ \\
\hline 26 & ND & ND & ND & $1.77 \pm 0.11$ & ND & ND & ND & ND & 0.003 & ND & ND & ND & ND & ND & $0.27 \pm 0.38$ \\
\hline 27 & ND & ND & ND & $1.21 \pm 0.05$ & ND & ND & ND & ND & ND & ND & ND & 0.007 & ND & ND & $0.237 \pm 0.001$ \\
\hline 28 & ND & ND & ND & 0.15 & ND & ND & ND & ND & ND & ND & ND & ND & ND & ND & $0.36 \pm 0.52$ \\
\hline 29 & ND & ND & ND & 0.18 & ND & ND & ND & ND & ND & ND & ND & ND & ND & ND & $0.76 \pm 0.89$ \\
\hline 30 & ND & ND & $\mathrm{ND}$ & $0.851 \pm 0.014$ & ND & ND & ND & ND & ND & ND & ND & ND & ND & ND & $5.86 \pm 0.09$ \\
\hline 31 & ND & ND & 0.007 & 0.38 & ND & ND & 0.036 & 0.036 & 0.008 & ND & ND & ND & ND & ND & $N D$ \\
\hline 32 & ND & ND & $0.129 \pm 0.013$ & 0.15 & 1.2 & 2.8 & ND & ND & 0.013 & 0.030 & 0.016 & ND & ND & ND & 0.010 \\
\hline 33 & ND & ND & $0.068 \pm 0.008$ & ND & ND & ND & 0.027 & ND & ND & ND & ND & ND & ND & 0.035 & ND \\
\hline 34 & ND & ND & $0.330 \pm 0.010$ & $1.07 \pm 0.51$ & $11.6 \pm 3.0$ & 5.6 & 0.017 & 0.042 & ND & $0.60 \pm 0.13$ & ND & ND & ND & ND & $3.54 \pm 0.44$ \\
\hline 35 & 0.061 & ND & $0.211 \pm 0.045$ & 0.17 & ND & 2.6 & ND & ND & ND & 0.061 & ND & ND & ND & ND & 0.036 \\
\hline
\end{tabular}




\section{Supplemental Material}

\begin{tabular}{|c|c|c|c|c|c|c|c|c|c|c|c|c|c|c|c|}
\hline$\#$ & $\mathrm{Cr}$ & Mn & Co & $\mathrm{Ni}$ & $\mathrm{Cu}$ & $\mathrm{Zn}$ & As & Mo & Cd & Sn & $\mathrm{Sb}$ & $\mathrm{Pt}$ & $\mathrm{Au}$ & $\mathrm{TI}$ & $\mathrm{Pb}$ \\
\hline 36 & ND & ND & $0.128 \pm 0.010$ & 0.07 & ND & 3.0 & ND & ND & ND & ND & ND & ND & ND & ND & $0.081 \pm 0.019$ \\
\hline 37 & ND & ND & $0.169 \pm 0.012$ & ND & ND & 3.8 & ND & ND & ND & ND & ND & ND & ND & ND & $0.069 \pm 0.001$ \\
\hline 38 & ND & ND & $0.397 \pm 0.060$ & 0.07 & ND & 4.5 & ND & ND & ND & 0.051 & ND & ND & ND & ND & $0.063 \pm 0.015$ \\
\hline 39 & ND & ND & $0.214 \pm 0.031$ & ND & 0.4 & ND & ND & ND & ND & ND & ND & ND & ND & ND & $0.062 \pm 0.006$ \\
\hline 40 & ND & ND & $0.156 \pm 0.018$ & 0.05 & ND & ND & ND & ND & ND & ND & ND & ND & ND & ND & 0.026 \\
\hline 41 & 0.052 & ND & $0.118 \pm 0.013$ & 0.12 & 0.5 & 3.2 & ND & ND & ND & ND & ND & ND & ND & ND & $0.41 \pm 0.90$ \\
\hline 42 & ND & ND & ND & ND & ND & ND & 0.007 & ND & ND & ND & ND & ND & ND & ND & ND \\
\hline 43 & ND & ND & $0.055 \pm 0.001$ & 0.05 & ND & 1.9 & ND & ND & ND & ND & ND & ND & ND & ND & $0.071 \pm 0.000$ \\
\hline 44 & ND & ND & $0.172 \pm 0.056$ & 0.05 & ND & 2.4 & ND & ND & ND & ND & ND & ND & ND & ND & $0.101 \pm 0.030$ \\
\hline 46 & ND & ND & $0.306 \pm 0.007$ & 0.15 & 0.4 & 9.7 & ND & ND & ND & 0.180 & ND & ND & ND & ND & $0.180 \pm 0.089$ \\
\hline 47 & ND & ND & $0.090 \pm 0.004$ & ND & ND & ND & ND & ND & ND & ND & ND & ND & ND & ND & $1.0 \pm 2.7$ \\
\hline 48 & ND & ND & $0.053 \pm 0.005$ & ND & ND & ND & ND & ND & ND & ND & ND & ND & ND & ND & 0.014 \\
\hline 49 & ND & ND & $0.052 \pm 0.025$ & 0.09 & ND & ND & ND & ND & ND & ND & ND & ND & ND & ND & 0.018 \\
\hline 50 & ND & ND & ND & ND & ND & ND & ND & ND & ND & ND & ND & ND & ND & ND & $\mathrm{ND}$ \\
\hline 51 & ND & ND & $0.019 \pm 0.003$ & 0.14 & ND & ND & ND & ND & ND & ND & ND & ND & ND & ND & 0.037 \\
\hline 52 & ND & 0.012 & $0.582 \pm 0.029$ & $1.91 \pm 0.54$ & $18.9 \pm 8.0$ & $19.3 \pm 5.2$ & 0.009 & ND & 0.002 & $0.74 \pm 0.26$ & ND & ND & 0.043 & ND & $2.59 \pm 0.39$ \\
\hline 53 & 0.041 & ND & $0.968 \pm 0.048$ & $5.46 \pm 0.16$ & $6.53 \pm 0.35$ & $14.9 \pm 0.3$ & 0.010 & ND & ND & $1.07 \pm 0.22$ & ND & ND & ND & ND & $2.49 \pm 0.08$ \\
\hline 54 & 0.038 & 0.010 & $0.822 \pm 0.007$ & $4.8 \pm 2.2$ & $18 \pm 13$ & $23 \pm 10$ & 0.010 & ND & 0.002 & $0.99 \pm 0.44$ & ND & ND & ND & ND & $3.60 \pm 0.19$ \\
\hline
\end{tabular}

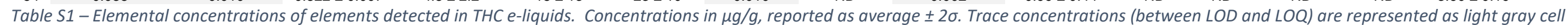
shading with $\leq 2$ significant figures with no reported uncertainty. 


\section{Supplemental Material}

\begin{tabular}{|c|c|c|c|c|c|c|c|c|c|c|c|c|c|c|}
\hline \# & $\mathrm{Cr}$ & Mn & Co & $\mathrm{Ni}$ & $\mathrm{Cu}$ & $\mathrm{Zn}$ & As & Mo & Cd & Sn & $\mathrm{Sb}$ & $\mathrm{Pt}$ & $\mathrm{Au}$ & $\mathrm{Pb}$ \\
\hline A & ND & 0.018 & 0.004 & ND & ND & ND & 0.015 & ND & ND & ND & ND & ND & ND & 0.008 \\
\hline B & $0.486 \pm 0.020$ & $0.181 \pm 0.015$ & $0.160 \pm 0.005$ & $34.6 \pm 1.7$ & $7.23 \pm 0.29$ & 7.48 & 0.012 & ND & ND & 0.20 & 0.017 & ND & $2.27 \pm 0.12$ & ND \\
\hline C & 0.25 & $0.142 \pm 0.005$ & $0.038 \pm 0.007$ & $6.53 \pm 0.54$ & 0.95 & 3.58 & ND & ND & ND & 0.037 & ND & ND & ND & 0.027 \\
\hline D & ND & ND & ND & $0.65 \pm 0.10$ & 0.60 & ND & ND & ND & ND & ND & ND & ND & ND & ND \\
\hline E & ND & ND & 0.005 & $0.721 \pm 0.064$ & $1.44 \pm 0.10$ & ND & ND & 0.026 & ND & ND & ND & 0.014 & 0.020 & ND \\
\hline$F$ & ND & 0.017 & 0.003 & 0.15 & ND & ND & ND & ND & ND & ND & ND & ND & ND & ND \\
\hline G & ND & 0.011 & 0.002 & ND & 0.39 & ND & ND & ND & ND & 0.066 & ND & ND & ND & ND \\
\hline H & 0.21 & $0.193 \pm 0.040$ & $0.718 \pm 0.009$ & $40.4 \pm 3.2$ & $116 \pm 8$ & $79.2 \pm 5.1$ & 0.011 & $0.356 \pm 0.059$ & 0.008 & $2.91 \pm 0.73$ & 0.032 & ND & ND & $6.17 \pm 0.09$ \\
\hline I & ND & 0.013 & 0.004 & 0.13 & ND & 1.83 & ND & ND & ND & ND & ND & 0.033 & ND & ND \\
\hline J & ND & 0.013 & 0.003 & 0.07 & ND & 2.27 & ND & ND & ND & ND & ND & $0.040 \pm 0.006$ & ND & ND \\
\hline K & ND & 0.075 & 0.009 & $1.73 \pm 0.12$ & $33.8 \pm 1.4$ & $24.6 \pm 1.1$ & ND & ND & 0.006 & 0.18 & ND & ND & ND & $4.17 \pm 0.19$ \\
\hline L & $1.28 \pm 0.04$ & 0.020 & 0.011 & $21.2 \pm 0.7$ & $105 \pm 1$ & $64.5 \pm 2.1$ & ND & 0.026 & ND & $0.594 \pm 0.050$ & 0.019 & ND & 0.020 & $3.41 \pm 0.03$ \\
\hline M & ND & 0.013 & 0.005 & 0.077 & ND & ND & ND & ND & ND & ND & ND & ND & ND & ND \\
\hline
\end{tabular}

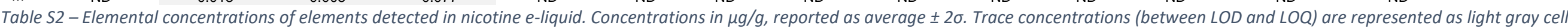
shading with $\leq 2$ significant figures with no reported uncertainty. 


\section{Supplemental Material}

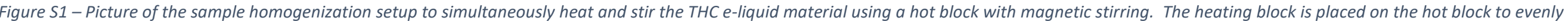

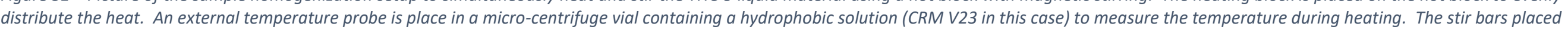

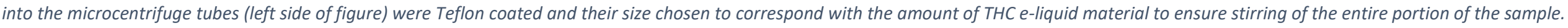

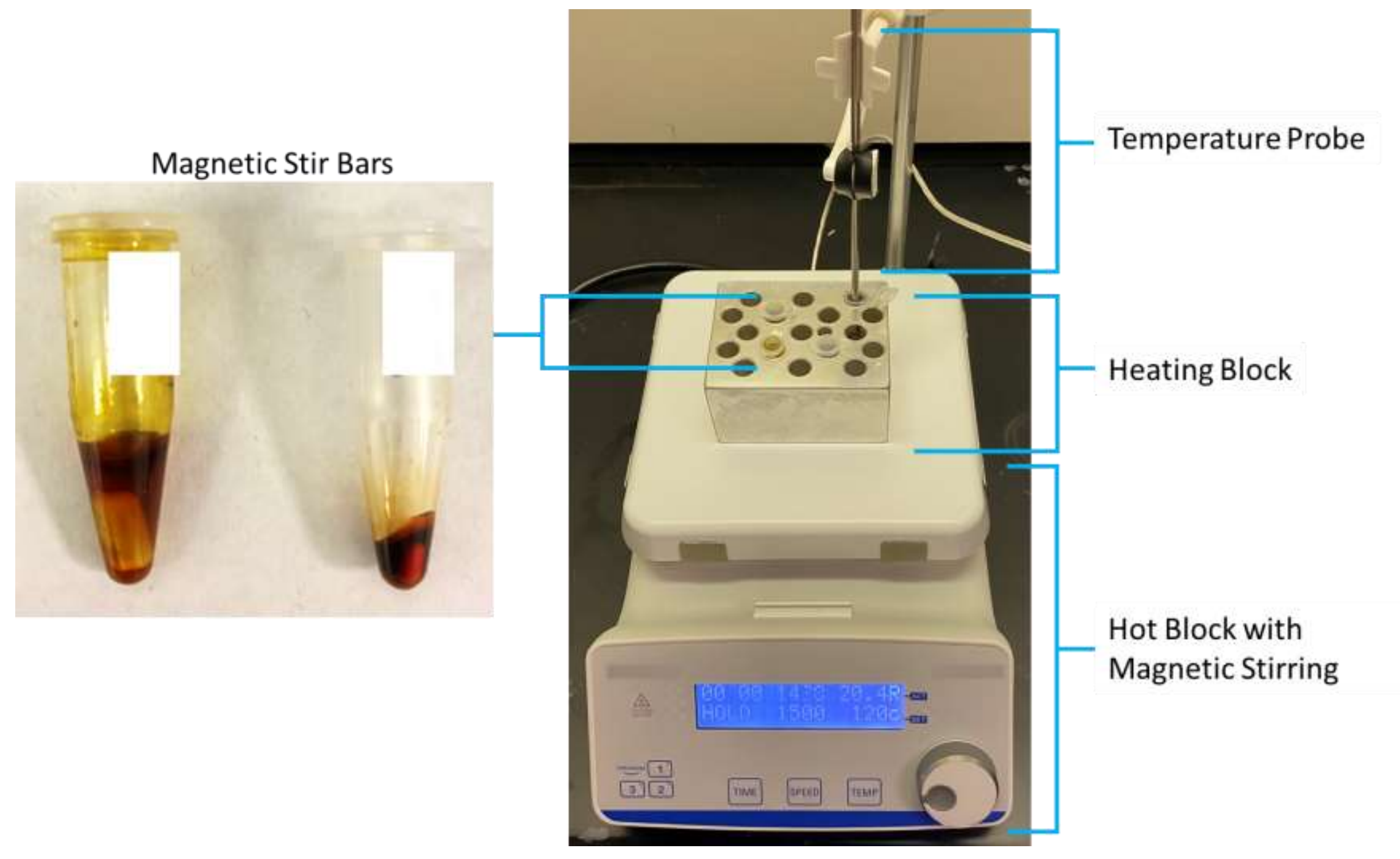

